\title{
Effect of Oxidation of Cobalt-Based Nanowires on NMR Spin-Lattice Relaxation
}

\author{
H. De Riedmatten, V. Scarani, and J.-P. Ansermet \\ Institut de Physique Expérimentale, Ecole Polytechnique Fédérale de Lausanne, \\ Lausanne, Switzerland
}

Received July 19, 1999; revised August 2, 2000

\begin{abstract}
Nuclear spin-lattice relaxation measurements were performed on Co-based magnetic nanowires, in zero field and in $1 \mathrm{~T}$ applied field. A measurement method is developped that allows a confident interpretation of the experimental data. An enhanced relaxation due to a thermally activated phenomenon associated to oxidation is reported for the first time in the nuclear magnetic resonance of ferromagnetic systems.
\end{abstract}

\section{Introduction}

In the last decade, the need for characterizing the properties of magnetic nanostructures implied a revival of interest in the nuclear magnetic resonance (NMR) of ferromagnetic systems $[1,2]$. The spin-lattice relaxation (SLR) time $T_{1}$ might appear as a good parameter for monitoring spin waves. However, this intuitive statement turns out to be far from obvious: the role of electronic spin waves on $T_{1}$ has often been invoked but never directly demonstrated. For nuclei in domains, the controversy dates back to Weger [3] and Moriya [4] and has not been settled according to more recent work (see, e.g., $[5,6]$ ). Moreover, the very measurement of SLR has peculiar features, like the dependence of relaxation on the applied radio-frequency (rf) field [7], that raise serious problems about method and interpretation. At the beginning of NMR studies of ferromagnetic samples, some authors tried to describe the SLR mechanism for nuclei in domain walls (DW) $[3,8,9]$. Recently, Dho and Lee [10] resumed this controversy. Except Weger's work [3] (whose data and interpretation have been seriously questioned $[4,6])$, all these studies concern ${ }^{57} \mathrm{Fe}$ NMR. In spite of the relative simplicity of the NMR of this isotope, a comprehensive picture of the relaxation mechanisms is still lacking.

It is obvious that ${ }^{59} \mathrm{Co}$ (with its spin $7 / 2$ and its many possible crystallographic structures) is not the suitable isotope to explore the fundamental mechanisms 
governing the NMR of ferromagnets; in particular, the zero-field value of $T_{1}$ of ${ }^{59} \mathrm{Co}$ has been considered as ill-defined [11]. In our case, the choice of this nucleus is linked to the study of spin-dependent transport. We describe a suitable calibration protocol allowing a consistent interpretation of $T_{1}$ even in the zero-field situation; and we show that by measuring $T_{1}$ between $20 \mathrm{~K}$ and room temperature we can monitor the oxidation process of cobalt.

\section{Samples}

Samples under study are cobalt-based magnetic nanowires grown by electrodeposition in a polycarbonate membrane [12]. Each sample consists of about $10^{10}$ wires. One of the advantages of such templates is the possibility of detecting a room temperature signal. In this work we have studied arrays of Co wires and multilayered $\mathrm{Co} / \mathrm{Cu}$ wires, both grown with a bath containing a $\mathrm{Cu}$ salt. The facecentered cubic (fcc) structure is dominating even in the case of homogeneous wires because of the presence of the $\mathrm{Cu}$ impurities codeposited with $\mathrm{Co}(15 \%$ in volume, in the form of clusters of few tenths of atoms) [13]. The length of the wires is $6 \mu \mathrm{m}$, the mean diameter is $80 \mathrm{~nm}$; the skin depth being typically longer (about $1 \mu \mathrm{m}$ ), we detect an NMR signal from the whole sample. The picture arising from the experimental data leads to the conclusion that we observe nuclei in domains $[14,15]$. In multilayers, the $\mathrm{Co}$ and $\mathrm{Cu}$ layers had the same thickness. Data are reported for layer thicknesses of $6,21,30$ and $48 \mathrm{~nm}$ (an error of $\pm 0.5 \mathrm{~nm}$ was determined on the statistics of growth times).

In our samples, the hyperfine field responsible for the Zeeman splitting of the nuclear spin levels cannot be considered as perpendicular to the applied if field $B_{1}$. In the literature, some discussion is given about the orientation of the global electronic magnetization with respect to the wires axis [16]. However, the absence of texture evidenced by X-ray measurements in our wires shows that this local magnetization has no preferred direction [13].

\section{Measurement of $T_{1}$}

The spectral line of bulk ${ }^{59} \mathrm{Co}$ in ferromagnetic samples has a width of about $20 \mathrm{MHz}$. When temperature is decreased from 300 to $20 \mathrm{~K}$, peaks shift to higher frequencies by an amount of about $4 \mathrm{MHz}$. We worked with a nonresonant probehead in order to have the same applied rf field over the whole frequency range. By measuring the voltage across the coil, we estimated $B_{1} \approx 0.5 \mathrm{G}$.

For the SLR measurements, we used a pulse sequence composed, as usual, of a saturating sequence that destroys nuclear magnetization, followed after a time $t$ by a spin-echo sequence $\tau$-wait- $\tau$ to detect the signal. The SLR curve is obtained by plotting the integral of the echo signal as a function of $t$. The singleexponential fit was always found in satisfactory agreement with the data. Since the hyperfine field has no preferred direction with respect to $B_{1}$, a special saturation sequence was used. It consisted of four long pulses, whose length is similar 
to the measured $T_{2}(20-30 \mu \mathrm{s})$. During these pulses, the nuclear magnetization nutates, and coherence is lost in the plane normal to the effective field. By applying these pulses in different directions, we obtain a satisfactory destruction of the nuclear signal.

Some unwanted mechanisms must be ruled out in order to interprete the signal recovery curve as a SLR. Cross-relaxation cannot be excluded a priori. The inhomogeneous broadening of the spectral line is enormous. This suggests that cross polarization is weak; but indirect coupling in ferromagnets is long-range. The influence of cross polarization has been ruled out by saturating the nuclear magnetization on a wide frequency range while detecting the spin-echo signal on a much narrower range. In our case, the length of the pulses and the spectrometer parameters impose a detection range of about $2 \mathrm{MHz}$. We used for the saturation sequence a second frequency generator with frequency modulation, and we obtained a saturation range of about $8 \mathrm{MHz}$ (Fig. 1). When the frequency range of saturation was reduced to about $1 \mathrm{MHz}$, the observed recovery rate was almost twice as high, a probable signature of cross-relaxation. Another spurious effect could be the selective irradiation of some quadrupolar transitions. This was excluded with a saturation range of $8 \mathrm{MHz}$, since quadrupolar splitting is known to be about $200 \mathrm{kHz}$ for Co in the hexagonal close-packed (hcp) phase [17, 18].

Therefore, all SLR data in this work were obtained by saturating the magnetization on a range of $8 \mathrm{MHz}$. We still had to tackle the problem of SLR measurements specific of ferromagnetic samples: the dependence of the measured SLR rate on the intensity of $B_{1}$. We show that this dependence comes from a distribution of the enhancement factor $\eta$, a factor that is proportional to the projection of the hyperfine field on the direction of $B_{1}$ and depends on the details of

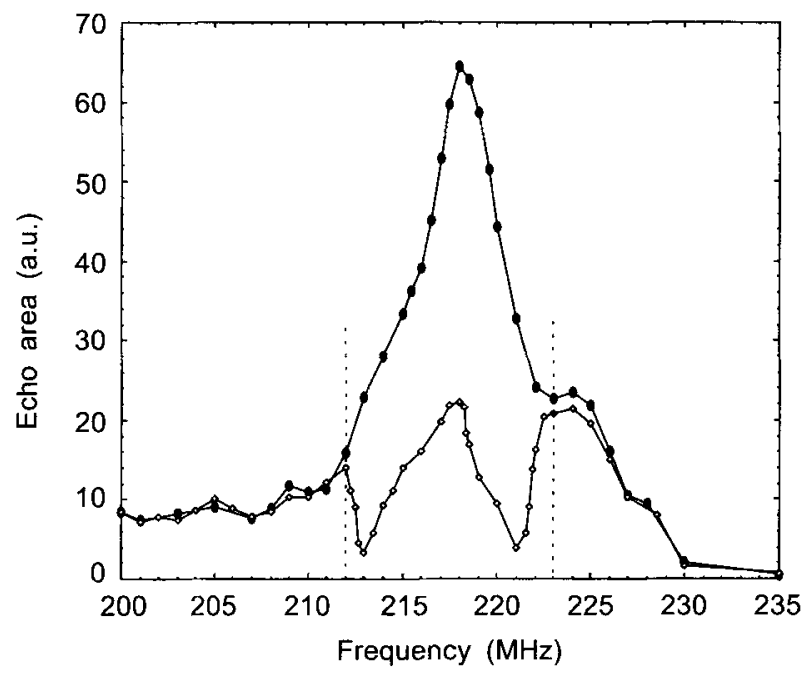

Fig. 1. Pointwise reconstruction of the spectral line of homogeneous $\mathrm{Co}+15 \% \mathrm{Cu}$ nanowires at $80 \mathrm{~K}$, recorded $200 \mathrm{~ns}(\mathrm{O})$ and $20 \mathrm{~ms}(\bullet)$ after the saturation sequence. 
domain-wall dynamics $[5,19,20]$. Given $B_{1}$ and a pulse length of the echo sequence $\tau$, a nuclear spin feeling an enhancement factor $\eta$ rotates by

$$
\theta\left(B_{1}, \tau, \eta\right)=(I+1 / 2) \gamma B_{1} \tau \eta,
$$

where the factor $I+1 / 2$ appears since we are dealing with a quadrupolar nucleus [15]. The observed signal comes mainly from those nuclei for which $\theta\left(B_{1}, \tau, \eta\right)=$ $2 \pi / 3$, since we are using a $\tau$-wait- $\tau$ echo sequence. The echo integral is actually found to scale with $B_{1} \tau$, as expected. And also the value $1 / T_{1}$ obtained by varying both the intensity of the $\mathrm{rf}$ field $B_{1}$ and the pulse length $\tau$ has been found to scale with $B_{1} \tau$, on several samples at various temperatures. This demonstrates that the $B_{1}$-dependence of $1 / T_{1}$ is actually a dependence on $\eta$, as announced. We define $\eta_{0}=(2 \pi / 3)\left[4 \gamma\left(B_{1} \tau\right)_{\max }\right]^{-1}$, where $\left(B_{1} \tau\right)_{\max }$ is the value of $B_{1} \tau$ that gives the maximum value of the echo integral. $\eta_{0}$ was found to range between 60 and 200 in all our samples and for all temperatures.

We are now able to describe our protocol for SLR measurements and to stress the underlying physical hypothesis. First, at any temperature we draw the spectral line and select a peak. On this peak we perform the pulse calibration, and we select the pulse length giving the maximum signal that defines $\eta_{0}$ at any temperature. We make the fundamental (and reasonable) hypothesis that the spins giving the maximum signal are the same at all temperatures. We did not perform systematic SLR measurements on other values of the enhancement factor. (The study of the $\eta$-dependence of SLR was performed on the fcc peak of homogeneous wires at $80 \mathrm{~K}$. The dependence is approximately linear for $\pi / 6 \gamma B_{1} \tau<$ $500 \approx 3 \eta_{0}$. The extrapolated SLR rate in the $\eta \rightarrow 0$ limit is not zero. For higher values a limiting upper value of $1 / T_{1}$ is reached. This means that $3 \eta_{0}$ is the maximum enhancement factor in the studied sample: for low values of $B_{1} \tau$, actually no nuclear spin rotates by $\theta=2 \pi / 3$, and we detect the signal of the nuclei that have the maximum enhancement factor, but that have not yet undergone a $2 \pi / 3$ rotation.)

\section{Experimental Data}

When the sample grows old, a structural modification is observed: an hcp peak tends to develop, even for samples that presented no hcp feature when measured just after their fabrication (Fig. 2). This effect and oxidation are of course coexisting; however, we cannot conclude that oxidation is the cause of the structural modification: since hcp is the thermodynamically stable phase for $\mathrm{Co}$, it is possible that some spontaneous annealing on a nanoscale takes place.

The reported SLR measurements were always performed on the same peak, bulk fcc, since this peak was well-defined in all samples. No significant difference in $T_{1}$ was seen by measuring other peaks (hcp and first $\mathrm{Cu}$ neighbor) when present.

Figure 3 shows the temperature dependence of the SLR relaxation rate $1 / T_{1}$ for homogeneous wires, measured in zero field under different oxidation conditions. The first measurement was performed four weeks after the fabrication of 


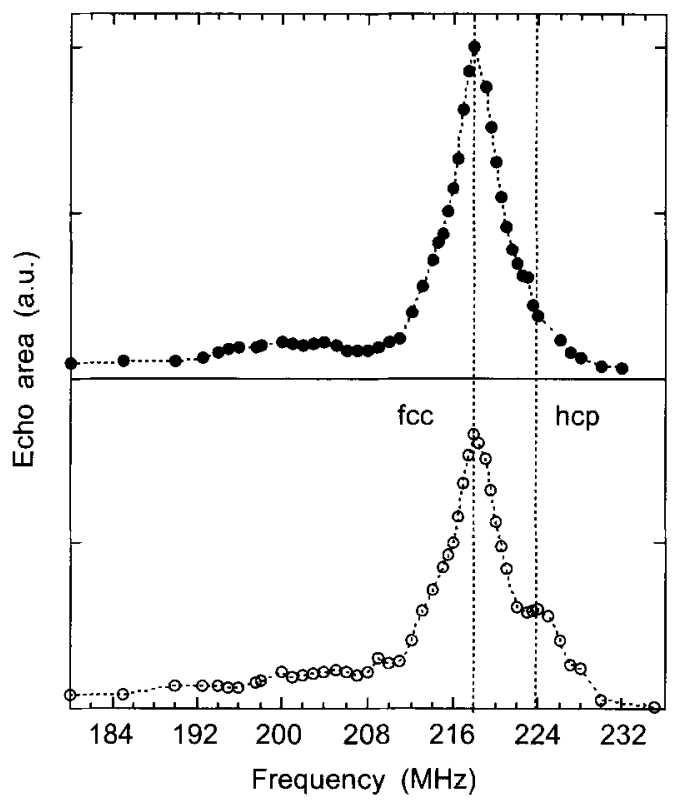

Fig. 2. Spectral lines for homogeneous $\mathrm{Co}+15 \% \mathrm{Cu}$ nanowires at $80 \mathrm{~K}$, before $(\bullet)$ and after $(0)$ oxidation in air. The emergence of an hcp peak is more probably the result of an annealing than a consequence of oxidation.

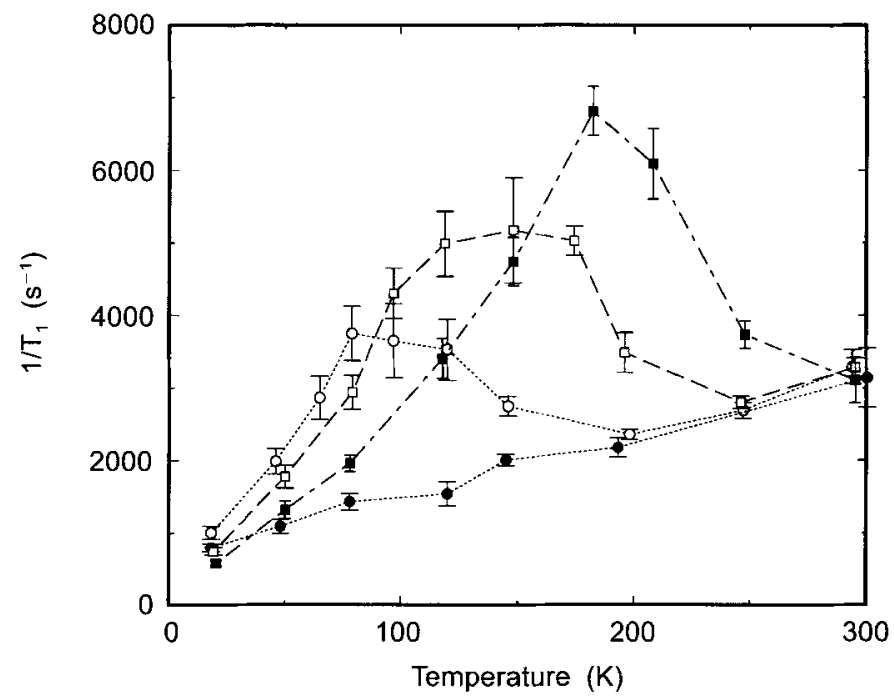

Fig. 3. $1 / T_{1}$ as a function of temperature in zero-field for homogeneous $\mathrm{Co}+15 \% \mathrm{Cu}$ nanowires, under increasing oxidation degrees ( nonoxidized; $O 1$ month in air; $48 \mathrm{~h}$ in oxygen at $100^{\circ} \mathrm{C}$; $\square 200$ more hours in oxygen at $100^{\circ} \mathrm{C}$ ). The lines are a guide for the eye. 
the sample. We observe a linear temperature dependence, with a slope $1 / T_{1} T=$ $8.1 \pm 0.41 / \mathrm{sK}$. The comparison with the literature is not easy because reported values range on two orders of magnitude [21]; our value is similar to the value of $13 \mathrm{l} / \mathrm{sK}$ found by Weger [3] and Jaccarino et al. [22]; and we could have doubled the value by selecting the nuclei having the highest enhancement instead of $\eta_{0}$. The absence of peculiar features due to oxidation is not astonishing since the wires are embedded in a polycarbonate membrane, and one must wait for oxygen to diffuse. The sample was then measured one month later, and a new feature, a peak, was observed in the $T_{1}(T)$ behavior. To confirm that this modification is due to oxidation, we accelerated the oxidation process by letting the sample for $48 \mathrm{~h}$ and then for further $200 \mathrm{~h}$ in an oxygen atmosphere at $100^{\circ} \mathrm{C}$. The peak was indeed found at higher temperatures. It was necessary to measure up to room temperature to have a correct interpretation of the $T_{1}(T)$ behavior; a measurement limited to $100 \mathrm{~K}$ would have led to an erroneous interpretation.

An applied field of $1 \mathrm{~T}$ reduces but does not suppress the peak. In this sample, the enhancement factor for the selected spins $\eta_{0}$ shows the same temperature behavior as the SLR rate; however, this feature is not universal for all samples. The peak in the SLR rate had already been observed in previous measurements on other samples, performed without pulse calibration: the calibration does not "generate" the peak, it simply allows a confident interpretation of the data.

The same behavior for the temperature dependence of $1 / T_{1}$ was found for $\mathrm{Co} / \mathrm{Cu}$ multilayered nanowires oxidized in air. In zero external field, the slope of the linear term was approximately twice as steep in multilayers as in homogeneous wires; in a $1 \mathrm{~T}$ field, this difference is suppressed [14]. Once the linear term is subtracted from $1 / T_{1}(T)$, we are left with the peaks, which in multilayers are centered at higher temperatures compared to homogeneous wires oxidated in air.

\section{Conclusion}

A consistent measurement method of $T_{1}$ in zero-field ${ }^{59} \mathrm{Co}$ NMR of nanosized ferromagnetic samples has been developed, that allows a confident measurement of this parameter. A new relaxation mechanism has been seen: its features are typical of a thermally activated phenomenon [14]; to our knowledge, such a mechanism has never been reported for SLR in ferromagnetic systems.

\section{References}

1. Panissod P. in: Frontiers in Magnetism of Reduced Dimension Systems (Baryakhtar V.G., Wigen P.E., Lesnik N.A., eds.). Dodrecht: Kluwer Academic Press 1998.

2. de Gronckel H.A.M., de Jonge W.J.M. in: Magnetic Multilayers (Bennett L.H., Watson R.E., eds.). Singapore: World Scientific 1994.

3. Weger M.: Phys. Rev. 128, 1505-15I1 (1962)

4. Moriya T.: J. Phys. Soc. Jpn. 19, 681-687 (1964)

5. Turrell B.G.: Hyperfine Interact. 7, 429-448 (1980)

6. Bobek C., Dullenbacher R., Klein E.: Hyperfine Interact. 77, 327-342 (1993) 
7. Weisman I.D., Swartzendruber L.J., Bennett L.H. in: Nuclear Resonance in Metals. Measurement of Physical Properties, Part 2: Magnetic Properties and Mössbauer Effect (Passaglia E., ed.). New York: Wiley Interscience 1973.

8. Winter J.M.: Phys. Rev. 124, 452-459 (1961)

9. Stearns M.B.: Phys. Rev. 187, 648-656 (1969)

10. Dho J., Lee S.: Phys. Rev. B 56, 7835-7838 (1997)

11. Kaplan N., Jaccarino V., Lewis R.T.: J. Appl. Phys. 39, 500-503 (1968)

12. Blondel A., Meier J.P., Doudin B., Ansermet J.-P.: Appl. Phys. Lett. 65, 3019-3021 (1994)

13. Scarani V., Doudin B., Ansermet J.-P.: J. Magn. Magn. Mat. 205, 241-248 (1999)

14. ScaraniV., de Riedmatten H., Ansermet J.-P.: Appl. Phys. Lett. 76, 903-905 (2000)

15. Scarani V.: Ph. D. Thesis, Ecole Polytechnique Fédérale de Lausanne, Lausanne, Switzerland 2000.

16. Ferré R., Ounadjela K., George J.M., Piraux L., Dubois S.: Phys. Rev. B 56, 14066-14075 (1997)

17. Riedi P.C., Scurlock R.G.: Phys. Lett. A 24, 42-43 (1967)

18. Fekete D., Boasson H., Grayevski A., Zevin V., Kaplan N.: Phys. Rev. B 17, 347-354 (1978)

19. Turov E.A., Petrov M.P.: Nuclear Magnetic Resonance in Ferro- and Antiferromagnets: New York: Halsted 1972.

20. Stearns M.B.: Phys. Rev. 162, 496-509 (1967)

21. Stearns M.B. in: Landolt-Börnstein, Numerical Data and Functional Relationships in Science and Technology, New Series III/19a, p. 68. New York: Springer 1987.

22. Jaccarino V., Kaplan N., Walstedt R.E., Wernick J.H.: Phys. Lett. 23, 514-515 (1966)

Authors address: Jean-Philippe Ansermet, Institut de Physique Expérimentale, Ecole Polytechnique Fédérale de Lausanne, CH-1015 Lausanne, Switzerland 